\title{
HYPOGLYCEMIA ACTIVATES COMPENSATORY MECHANISM OF GLUCOSE METABOLISM OF BRAIN
}

\author{
Teréz Márián, ${ }^{1}$ L. Balkay, ${ }^{2}$ I. Fekete, ${ }^{1}$ Zs. Lengyel,,${ }^{1}$ G. Veress,${ }^{4}$ \\ O. ÉsiK, ${ }^{4}$ L. Trón ${ }^{1}$ and Z. KRASZNAi ${ }^{3 *}$ \\ ${ }^{1}$ Positron Emission Tomograph Centre, \\ ${ }^{2}$ Department of Neurology and \\ ${ }^{3}$ Biophysics and Cell Biology, University of Debrecen, \\ Medical and Health Science Center, Debrecen, Hungary \\ ${ }^{4}$ Department of Radiotherapy, National Institute of Oncology, Budapest, Hungary
}

(Received: January 27, 2000; accepted: October 20, 2000)

\begin{abstract}
The effect of plasma glucose concentration on the cerebral uptake of $\left[{ }^{18} \mathrm{~F}\right]$-fluorodeoxy-D-glucose (FDG) was studied in a broad concentration range in a rabbit brain model using dynamic FDG PET measurements. Hypoglycemic and hyperglycemic conditions were maintained by manipulating plasma glucose applying i.v. glucose or insulin load. FDG utilization (K) and cerebral glucose metabolic rate (CGMR) were evaluated in a plasma glucose concentration range between $0.5 \mathrm{mM}$ and $26 \mathrm{mM}$ from the kinetic constant $k_{1}, k_{2}, k_{3}$ obtained by the Sokoloff model of FDG accumulation. A decreasing set of standard FDG uptake values found with increasing blood glucose concentration was explained by competition between the plasma glucose and the radiopharmacon FDG. A similar trend was observed for the forward kinetic constants $k_{1}$, and $k_{3}$ in the entire concentration range studied. The same decreasing tendency of $k_{2}$ was of a smaller magnitude and was reverted at the lowest glucose concentrations where a pronounced decrease of this backward transport rate constant was detected. Our kinetic data indicate a modulation of the kinetics of carbohydrate metabolism by the blood glucose concentration and report on a special mechanism compensating for the low glucose supply under conditions of extremely low blood glucose level.
\end{abstract}

Keywords: Dynamic FDG PET - CGMR - hypoglycemia

\section{INTRODUCTION}

Glucose is a major substrate for brain energy metabolism, and a significant fraction of glucose metabolism is coupled to neuronal activity. It is of critical importance for normal function and resistance to injury in both the mature and developing brain [1, 21]. The plasma membranes of most mammalian cells contain one or more proteins, which transport glucose by facilitated diffusion. The principal glucose transporters in the brain are GLUT1 (present at high concentration at the blood brain barrier and astrocytes), GLUT3 isoforms expressed in neurones and GLUT5 in microglia [15, $16,28]$

\footnotetext{
*Corresponding author; E-mail: krasznai@jaguar.dote.hu
} 
Although brain glucose metabolism is basically phosphorylation limited [17] transport processes may become rate limiting under special conditions. Changes in the expression of GLUT 1 and GLUT 3 have been reported during postnatal development $[19,26]$, following transient focal ischemic injury [25] and under other special conditions $[4,15]$. Great attention has been given to the changes in the D-glucose transport activity in hyperglycemic or hypoglycemic state $[5,11,12,13,17,19$, $20,29]$, but this intensively studied issue still has a number of unsolved questions and it has resulted in controversial results as well. A substantial part of the related investigations has been based on detecting changes in protein and/or RNA synthesis or transporter protein degradation, but research on the putative glucose dependent regulation of the brain glucose metabolism is required.

PET, using $\left[{ }^{18} \mathrm{~F}\right]-2$-fluoro-2-deoxy-D-glucose (FDG), enables the in vivo measurement of regional glucose metabolism under physiological and pathological conditions alike. FDG utilisation can be quantitized by using compartmental analysis [22] allowing the processes comprising the glucose metabolism to be investigated in detail. In this study, we sum up about the relationship between the kinetic parameters of FDG accumulation in rabbit brain and the plasma glucose level in a broad concentration range using dynamic PET investigations. Data analysis has revealed a special regulatory mechanism activated by extremely low blood sugar concentrations.

\section{MATERIALS AND METHODS}

\section{Animals}

Rabbits of $2.7 \pm 0.5 \mathrm{~kg}$ (mean $\pm \mathrm{SD}, \mathrm{n}=17$ ) were used throughout the experiments. The animals were provided ad libitum access to food except for the last five-hour fasting period prior to the PET investigations. The experimental protocol was approved by the Laboratory Animal Care and Use Committee of University Medical School of Debrecen based on the Helsinki declaration. In every experimental group (control, hyper- and hypoglycemic group) at least 5 animals were used. The rabbits were anaesthetised with i.p. urethane $(0.5 \mathrm{~g} / \mathrm{kg})$ and alpha-chloralose $(50 \mathrm{mg} / \mathrm{kg})$. One femoral artery and one femoral vein were cannulated [7]. Glucose loading (40\% glucose) and insulin (Actrapid MC $40 \mathrm{IU} / \mathrm{ml}, \mathrm{NOVO}$ ) was applied i.v. to manipulate the plasma glucose concentration. Hyperglycemia was achieved applying a glucose load of 2-3 $\mathrm{ml} 40 \%$ glucose $/ \mathrm{kg}$ body weight, 120 minutes prior to the FDG PET study, followed by an another $5 \mathrm{ml}$ dose 90 minutes later. Hypoglycemia was induced applying 15-20 IU insulin (120 minutes prior to the PET scan) followed by another insulin administration (10 IU), 30 minutes prior to the FGD injection. Plasma glucose levels were measured before the glucose and insulin treatments and during the PET scans as indicated in Fig. 1. Two animals were twice subjected to dynamic PET investigations under different conditions. Rabbit No 1 was manipulated in the first experiment in a way to have $26 \mathrm{mM}$, the highest (in the whole set of measurements), blood glucose concentration and two days later, the same animal was investigated 
under extremely low blood glucose concentration (1.1 mM glucose). Rabbit No 2 was adjusted first to hypoglycemic condition ( $2 \mathrm{mM}$ blood glucose concentration) and two days later this animal was used as a control with $7.3 \mathrm{mM}$ blood glucose concentration.

Serial arterial blood sampling was performed in all cases to allow the kinetic analysis of FDG accumulation.

\section{PET scanning}

FDG was synthesised according to Hamacher et al. [6]. The radiochemical purity of ${ }^{18} \mathrm{FDG}$ was higher than $97 \%$, and the specific activity was close to $2 \mathrm{Ci} / \mu \mathrm{mol}$. PET scans were carried out using a GE 4096 whole body PET camera. The laboratory animals were intravenously given $2-4 \mathrm{mCi} F D G$ in $2 \mathrm{ml}$ physiologic salt solution as a 20-30 sec bolus. All PET measurements were of dynamic type with the number and length of the exposures as follows: $12 \times 30 \mathrm{sec}, 5 \times 1 \mathrm{~min}, 5 \times 3 \mathrm{~min}$, and $3 \times 5 \mathrm{~min}$. Data used for the construction of static images were collected between the 40th and the 60th min. Transmission scans obtained with a $\mathrm{Ge}^{68} / \mathrm{Ga}^{68}$ external rotating rod source have been used to correct for tissue attenuation.

The regional FDG uptake was expressed as the standardized uptake value (SUV) and was calculated as the ratio of the decay-corrected tissue concentration (in $\mathrm{mCi} / \mathrm{kg}$ ) and the injected dose per body weight (in $\mathrm{mCi} / \mathrm{kg}$ ).

Dynamic analysis of brain glucose metabolism was carried out using the three compartment Sokoloff's model of glucose accumulation [22] as modified by Phelps et al. [18] including the dephosphorylation rate constant of FDG. Kinetic rate constants $\left(k_{1}\right.$ is the rate constants of the glucose transport from the intravasal compartment to the intracellular compartment, $k_{2}$ is the rate constant of the reverse transport, $k_{3}$ and $k_{4}$ are the rate constants of the phosphorylation and dephosphorylation reaction, respectively) of the model were evaluated using the matrix representation of the model [8]. The rate of FDG utilization ( $K$, expressed in $1 /$ min units) and the cerebral glucose metabolic rate (CGMR expressed in $\mathrm{mg} / 100 \mathrm{~g} / \mathrm{min})$ were calculated as:

$$
\begin{gathered}
K=\frac{k_{1} k_{3}}{k_{2}+k_{3}} \\
C G M R=\frac{C_{p}}{L c} \frac{k_{1} k_{3}}{k_{2}+k_{3}}
\end{gathered}
$$

where $C_{p}$ denotes plasma glucose concentration $(\mathrm{mg} / 100 \mathrm{~g}), L c$ is the Lumped constant, [22] the numerical value of which was assumed to be 1 . 


\section{RESULTS}

The plasma glucose concentrations of the untreated animals (without glucose and insulin load) were in the range of 5-12 $\mathrm{mM}(7.86 \pm 1.5, \mathrm{n}=6)$. Administration of glucose brought about an immediate increase in the blood glucose concentration followed by a slower decrease. Applying a second glucose load we succeeded to maintain a relatively constant hyperglycemic level of glucose during the PET scan (Fig. 1). The manipulated plasma glucose level of this group of animals ranged from 13 to $26 \mathrm{mM}(20.7 \pm 5.3, \mathrm{n}=6)$. Our insulin administration protocol resulted in an abrupt decrease of the plasma glucose concentration prior to the PET scan (Fig. 1B) and was efficient in maintaining very fairly stable hypoglycemic glucose levels in the range between 0.5 and $2.6 \mathrm{mM}(1.66 \pm 0.84, \mathrm{n}=5)$ during the PET measurements. For a
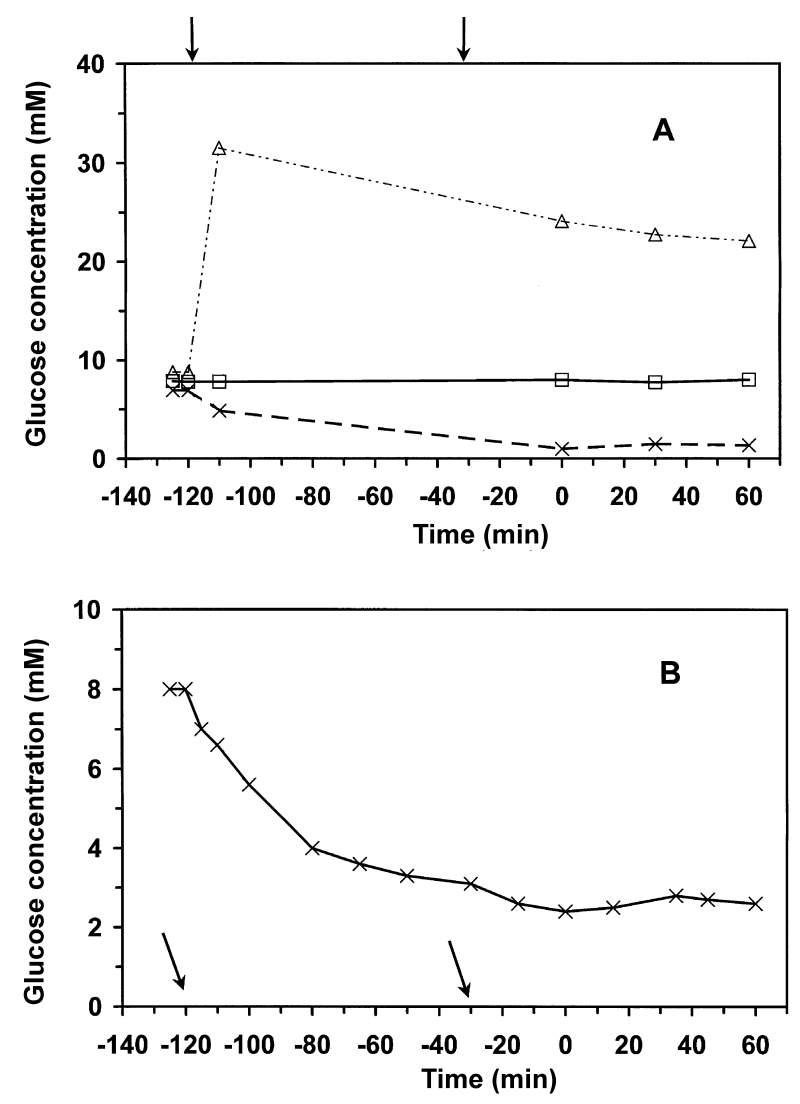

Fig. 1. A: Characteristic time dependence of plasma glucose concentration in a representative euglycemic $(\square)$, hypoglycemic $(\times)$ and hyperglycemic $(\triangle)$ animal. B: Time course of the plasma glucose concentration upon insulin treatment in a single experiment. Hypoglycemia was induced by i.v. administration of $20 \mathrm{IU}$ insulin 120 minutes prior to the FGD injection followed by another 10 IU insulin dose 90 minutes later. Arrows indicate time points of glucose and insulin administration 


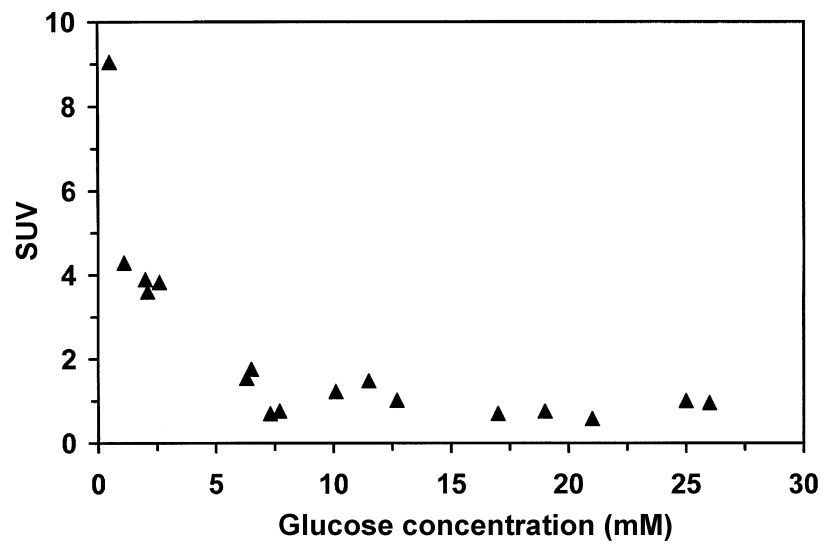

Fig. 2. Effect of plasma glucose concentration on the standard uptake value of FDG accumulation in rabbit brain

representative experiment a mean plasma glucose concentration of $2.6 \pm 0.15 \mathrm{mM}$ was obtained from data displayed in Fig. 1B averaged over the time of emission data collection.

The SUV were determined for the whole brain and plotted against plasma glucose concentration. The results displayed in Fig. 2 show a pronounced decrease of FDG accumulation with rising concentration of the glucose in the blood.

Results of the kinetic analysis are shown in Figs 3-5. Kinetic constant $k_{1}$ shows a monotonous decrease with growing blood glucose concentration in the whole range investigated (Fig. 3). Below $2 \mathrm{mM}$ plasma glucose level, the numerical value of this

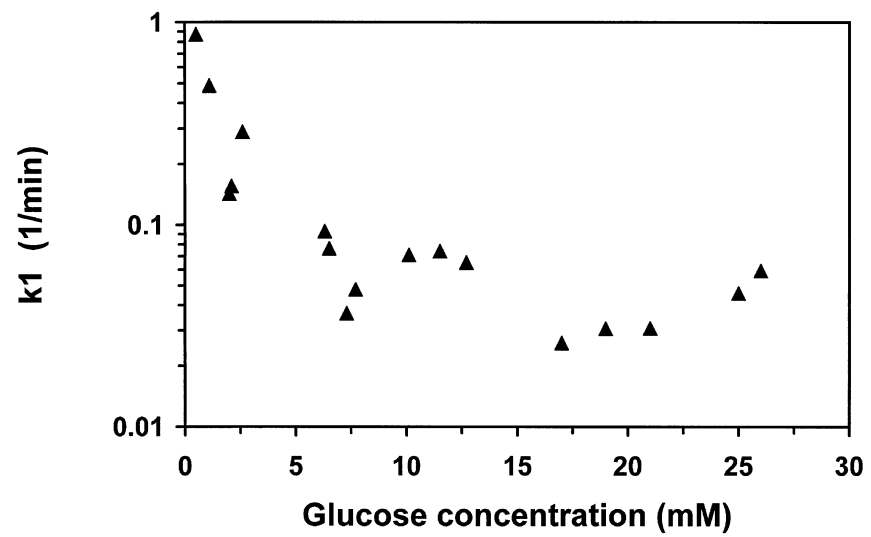

Fig. 3. Effect of plasma glucose concentration on the $k_{1}$ kinetic constant of glucose metabolism in the rabbit brain 


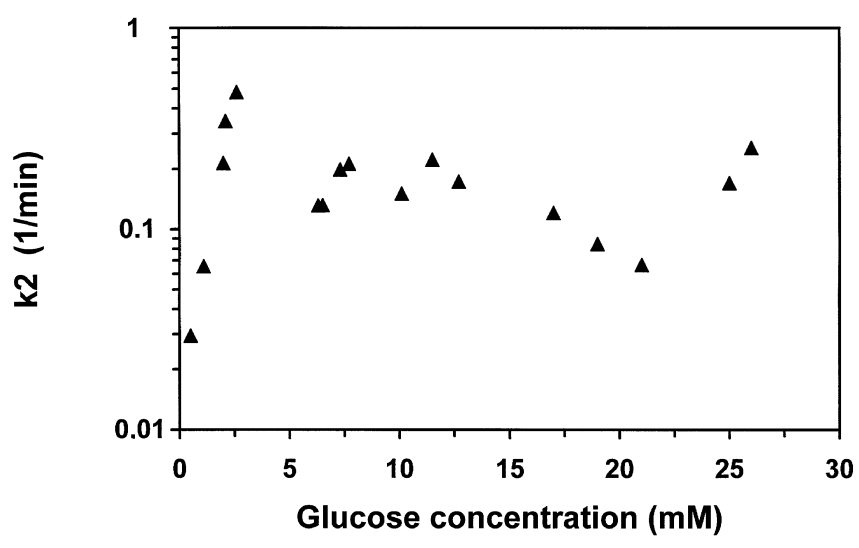

Fig. 4. Effect of plasma glucose concentration on the $k_{2}$ kinetic constant of glucose metabolism in the rabbit brain

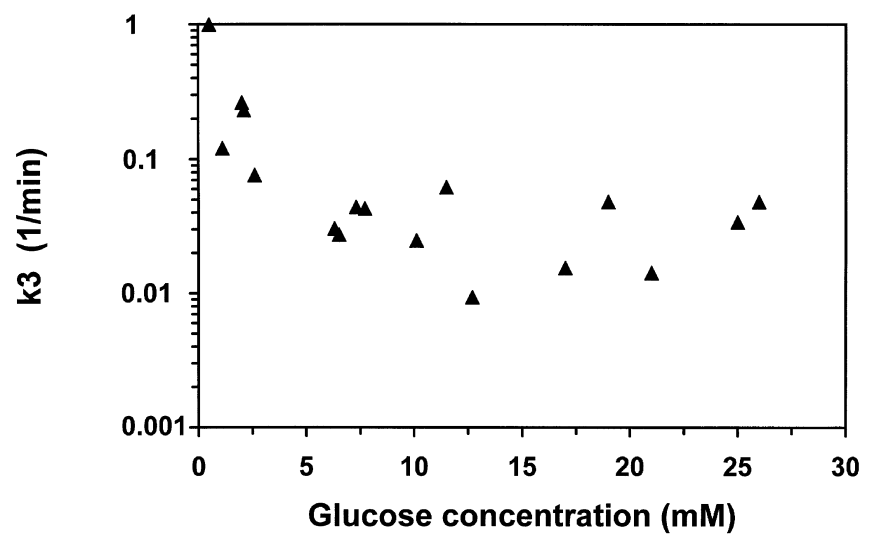

Fig. 5. Effect of plasma glucose concentration on the $k_{3}$ kinetic constant of glucose metabolism in the rabbit brain

constant increases by a factor of 8 . A similar tendency can also be observed in the change of $k_{3}$ (Fig. 5). The concentration dependence of these forward parameters is very similar to each other under euglycemic and hyperglycemic conditions. In contrast, although only a slight change was detected in $k_{2}$, characterizing the inverse transport of FDG from the intracellular compartment to the intravasal space, in the hyperglycemic and normoglycemic range, a remarkable decrease in its numerical value was detected at low plasma glucose levels (Fig. 4). At very low plasma glucose concentration $(0.5-1 \mathrm{mM})$ parameter $k_{2}$ approached zero which was accompanied by a 5-10 times increase in the values of $k_{1}$ and $k_{3}$. The kinetic constants of the hexokinase catalysed glucose phosphorylation decreased monotonously with increasing plasma glucose levels. 
Known values of $k_{1}, k_{2}$ and $k_{3}$ obtained from kinetic model calculations allowed the evaluation of the rate of FDG utilisation (Fig. 6) and the rate of cerebral glucose metabolism (Fig. 7). The former parameter showed a steadily decreasing tendency with the increase of the blood glucose level. Interestingly, the calculated CGMR vs plasma glucose concentration displayed minimum value in the physiological blood glucose concentration range and increased in both the hyperglycemic and hypoglycemic direction.

Two animals were reused in the experiments under conditions of different plasma glucose concentrations. With rabbit No. 1 hyperglycemic (extremely high level) con-

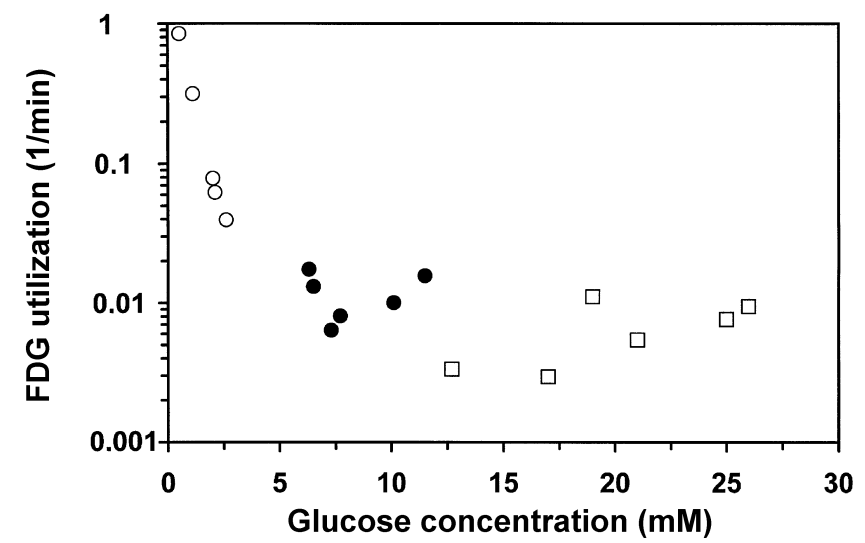

Fig. 6. Effect of plasma glucose concentration of the FDG utilization rate in the rabbit brain (euglycemic [(•), hypoglycemic $(\circ)$ and hyperglycemic $(\square)]$

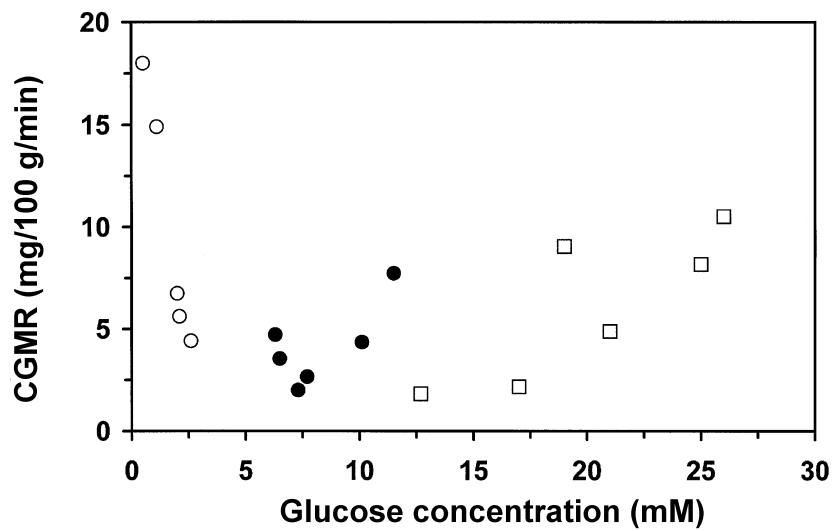

Fig. 7. Effect of plasma glucose concentration on the glucose metabolic rate (CGMR) in the rabbit brain [euglycemic (•), hypoglycemic (O) and hyperglycemic ( $\square)$ ] 
ditions were maintained first and in the experiment two days later FDG PET scan was completed on the same animal with hypoglycemic plasma glucose level. Rabbit No. 2 was subjected to the first measurement under hypoglycemic conditions and served as a control with physiological blood glucose concentration two days later in a second experiment. The results of the kinetic analyses of these measurements are summarised in Table 1. The results obtained with these rabbits fitted in well with the trend of other measurements in rabbits used in only a single run.

Table 1

Kinetic parameters of brain glucose metabolism on the rabbits each used under two different conditions of plasma glucose concentration

\begin{tabular}{lcccccc}
\hline & $\begin{array}{c}\text { Glucose, } \\
\mathrm{mM}\end{array}$ & $\begin{array}{c}K, \\
1 / \mathrm{min}\end{array}$ & $\begin{array}{c}k_{1}, \\
1 / \mathrm{min}\end{array}$ & $\begin{array}{c}k_{2}, \\
1 / \mathrm{min}\end{array}$ & $\begin{array}{c}k_{3}, \\
1 / \mathrm{min}\end{array}$ & $\begin{array}{c}\text { LGMR, } \\
\mathrm{mg} / 100 \mathrm{~g} / \mathrm{min}\end{array}$ \\
\hline Rabbit 1 & 26.0 & 0.00944 & 0.05944 & 0.25513 & 0.04817 & 10.5 \\
Rabbit 1 & 1.1 & 0.31600 & 0.48809 & 0.06545 & 0.12063 & 14.9 \\
Rabbit 2 & 2.0 & 0.07869 & 0.14270 & 0.21387 & 0.26280 & 6.74 \\
Rabbit 2 & 7.3 & 0.00639 & 0.03650 & 0.19870 & 0.04406 & 2 \\
\hline
\end{tabular}

\section{DISCUSSION}

Having special intention to carry out dynamic investigations of glucose metabolism in the widest possible range of glucose concentration, we have chosen a laboratory animal experimental system. The rabbit brain has been selected as an experimental system for this purpose as the rabbit model has proved to be suitable for PET investigations $[3,10]$. It has also been suggested that the evaluation of the kinetic constants is superior to the analysis of the FDG utilization rate as the former can reflect metabolic changes more specifically. The precise calculation of glucose metabolic rate assumes exact knowledge about the numerical value of the Lumped constant. As no $L c$ data are available on rabbit brain we have postulated $L c=1$. We assumed also that the value of the Lumped constant for rabbit brain does not change with the blood glucose concentration. This assumption has been made in analogy with the finding by Suda et al. [23] and Dienel et al. [2] who reported relatively stable Lumped constant for the rat brain in a wide range of plasma glucose concentration. Fairly stable normoglycemic and hypoglycemic plasma glucose concentrations were maintained during the time range of the PET measurements with the protocol outlined (Fig. 1). Manipulation of the blood glucose was carried out during the last 2 hours before the dynamic PET scans. This time interval proved to be long enough for the regulatory mechanisms to produce alterations in the kinetic parameters and it could also be compared with the reported ones during which the changes in glucose transporter expression (or translocation) and glucose transporter mRNA levels develop [29]. 
FDG uptake in rabbit brain tissue was inhibited by increased glucose concentration in the blood as it was seen from the inverse relationship between SUV values and plasma glucose level (Fig. 2). This finding was consistent with the competition between FDG and glucose. The extent of competition and the displacement of FDG by plasma glucose were determined by the $K_{m}$ values for these substrate molecules [22]. The relatively low $K_{m}$ values of GLUT1 and GLUT3 transporters resulted in a decline of the FDG uptake by the brain as the plasma glucose levels rose [17]. Moreover, FDG is a substrate analogue for hexokinase, thus both substrates compete for the binding/active site of this enzyme as well. Due to the competition of different level and other factors, the effect of blood glucose concentration on the FDG accumulation may be tissue dependent. Accordingly, the FDG uptake of different tissues was inhibited to different extent by high plasma glucose levels [9, 14, 24, 27, 28]. Glucose dependent changes in the individual rate constants of the FDG accumulation were studied in detail. Kinetic constant $k_{1}$, determining the transport rate from the intravasal space toward the extracellular and (non-phosphorylated) intracellular space, decreased monotonously with increasing glucose levels. Change in $k_{1}$ over the 5-26 mM glucose concentration range was surpassed by that over the $1-5 \mathrm{mM}$ range, the latter amounting to about an order of magnitude (Fig. 3). Glucose transport toward the tissues can become rate limiting at very low plasma glucose level and this may activate defined compensatory regulation mechanisms to increase the rate of this transport process.

Of interest is the finding that change in the reverse transport rate parallels that of $k_{1}$ only above $3 \mathrm{mM}$ glucose concentration but, below this threshold, an abrupt decrease of $k_{2}$ takes place. A similar change in the values of $k_{1}$ and $k_{2}$ would be consistent with a simple, glucose concentration induced change, also including the possibility of altered expression of the appropriate glucose transporter molecules or a translocation of these proteins. The change of opposite sign in these parameters means that these regulatory processes alone, even if they exist, cannot account for the detected behaviour of the transport constants. Allowing for a direct competition or an altered expression or even a translocation process, we still have to assume additional factors exerting different effect on forward and backward transport. One of the possibilities can be the appearance of a metabolite of a regulatory concentration in the hypoglycemic tissue environment, binding of which may result in altered transport characteristics of the transporter protein molecules but this assumption requires further experimentation.

The glucose concentration dependence of the FDG utilization rate is displayed in Fig. 6 as calculated from the numerical values of $k_{1}, k_{2}$ and $k_{3}$. This derived parameter shows the largest dynamic range with varying glucose concentration as it has contribution from all the three individual rate constants. It is interesting to note that the slight individual increase in the $k_{1}, k_{2}$ and $k_{3}$ parameters at the highest glucose levels results in a pronounced increase of the CGMR in this concentration range (Fig. 7). The rising trend of CGMR at the lowest blood glucose levels tested reports on an overcompensation of the loss in CGMR expected at very low blood glucose level by changing the molecular parameters of the involved elementary processes. 
In conclusion this study demonstrates that FDG uptake decreases in rabbit brain in animals with elevated blood glucose concentration. The reduced SUV values are in accord with the diminished numerical values of $k_{1}$, and $k_{3}$ in the studied blood glucose concentration range. Kinetic constant $k_{2}$ changes in a very similar way except in the lowest, $1 \mathrm{mM}-3 \mathrm{mM}$, concentration range. The observed changes are explained by a direct competition between FDG and glucose as well as an additional special compensatory mechanism.

\section{ACKNOWLEDGEMENT}

This study was partly supported by FKFP 1333/97, ETT 349/96, 362/96 and ETT 10/104/2000.

\section{REFERENCES}

1. Aller, B. C., Ehmann, S., Gilman-Sachs, A., Snyder, K. A. (1997) Flow cytometric analysis of glucose transport by rat brain cells. Cytometry 27, 262-268.

2. Dienel, G. A., Cruz, N. F., Mori, K., Holden, J., Sokoloff, L. (1991) Direct measurements of the $\gamma$ of the lumped constant of the deoxyglucose method in rat brain: determination of $\gamma$ and lumped constant from tissue glucose concentration or equilibrium tissue: plasma distribution ratio for methylglucose. J. Cereb. Blood Flow Metab. 11, 25-34.

3. Eng, H. L., DeLapaz, R. L., Frankel, K. A., Poljak, A., Phillips, M. H., Brennan, K. M., Woodruff, K. H., Valk, P. E., Steinberg, G. K., Fabrikant, J. I. (1991) MRI and PET delayed heavy-ion radiation injury in the rabbit brain. J. Radiation Oncology Biol. Phys. 20, 689-696.

4. Gamelli, R. L., Liu, H., He, L. K., Hoffmann, C. A. (1994) Alterations of glucose transporter mRNA and protein levels in brain following thermal injury and sepsis in mice. Shock 1, 395-400.

5. Germinario, R. J., Michaelidou, A. (1986) Hexose transport after glucose refeeding of glucosestarved human fibroblasts: 1 . The effects of tunicamycin and cycloheximide. 2. Insulin binding and action. Biochem Biophys Res. Commun 140, 844-849.

6. Hamacher, K., Coenen, H. H., Stöcklin, G. (1986) Efficient stereospecific synthesis of no-carrieradded2-( $\left.{ }^{18} \mathrm{~F}\right)$-Fluoro-2-Deoxy-D-Glucose using aminopolyether supported nucleophilic substitution. J. Nucl. Med. 27, 235-238.

7. Hegedús, K., Keresztes, T., Fekete, I., Molnár, L. (1997) Effect of i.v. dipyridamol on cerebral blood flow, blood pressure, plasma adenosine and cAMP levels in rabbits. J. Neuro. Sci . 148, 153-157.

8. Hoh, C. K., Dahlbom, M., Hawkins, R. A., Glaspy, J. A., Yao, W. J., Lee, S. J., Maddahi, J., Phelps, M. E. (1994) Basic principles of positron emission tomography in oncology: quantification and whole body techniques. Wien Klin. Wochenschr. 106, 496-504.

9. Ishizu, K., Nishizawa, S., Yonekura, Y., Sadato, N., Magata, Y., Tamaki, N., Tsuchida, T., Okazawa, H., Miyatake, S., Ishikawa, M., Kikuchi, H., Konishi, J. (1994) Effects of hyperglycemia on FDG uptake in human brain and glioma. J. Nucl. Med. 35, 1104-1109.

10. Ishiwata, K., Sakiyama, Y., Sakiyama, T., Shimada, J., Toyama, H., Oda, K., Suzuki, F., Senda, M. (1997) Myocardial adenosine $\mathrm{A}_{2 \mathrm{a}}$ receptor imaging of rabbit by PET with $\left[{ }^{11} \mathrm{C}\right] \mathrm{KF} 17837$. Ann. Nucl. Med. 3, 219-225.

11. Knuuti, J., Nuutila, P., Ruotsalainen, U., Saraste, M., Harkonen, R., Ahonen, A., Teras, M., Haaparanta, M., Wegelius, U., Haapanen, A. (1992) Euglicemic hyperinsulinemic clamp and oral glucose load in stimulating myocardial glucose utilization during positron emission tomography. $J$. Nucl. Med. 33, 1255-1262.

12. Kubota, K., Kubota, R., Yamada, S., Tada, M., Takahashi, T., Iwata, R. (1996) Re-evalution of myocardial FDG uptake in hyperglycemia. J. Nucl. Med. 37, 1713-1717. 
13. Lemmon, S. K., Sens, D. A., Buse, M. G. (1985) Insulin stimulation of glucose transport and metabolism in a human Wilms' tumor-derived myoblast-like cell line: modulation of hormone effects by glucose deprivation. J. Cell Physiol. 125, 456-464.

14. Lindholm, P., Minn, H., Leskinen-Kallio, S., Bergman, J., Ruotsalainen, U., Joensuu, H. (1993) Influence of the blood glucose concentration on FDG uptake in cancer-A PET study. J. Nucl. Med. $34,1-6$.

15. Maher, F., Vannucci, S. J., Simpson, I. A. (1994) Glucose transporter proteins in brain. FASEB J. 8, 1003-1011.

16. Nishioka, T., Oda, Y., Seino, Y., Yamamoto, T., Inagaki, N., Yano, H., Imura, H., Shigemoto, R., Kikuchi, H. (1992) Distribution of the glucose transporters in human brain tumors. Cancer Research 52, 3972-3979.

17. Pelligrini, D. A., LaManna, J. C., Duckrow, R. B., Bryan, R. M. Jr., Harik, S. I. (1992) Hyperglycemia and blood-brain barrier glucose transport. J. Cerebr. Blood Flow Metab. 12, 887-899.

18. Phelps, M. E., Huang, S. C., Hoffman, E. J., Selin, C., Sokoloff, L., Kuhl, D. E. (1979) Tomographic measurement of local cerebral glucose metabolic rate in humans with 2-[F-18]fluoro-2-deoxy-D-glucose: validation of Method. Ann. Neurol. 6, 371-388.

19. Sadiq, F., Holtzclaw, L., Chundu, K., Muzzafar, A., Devaskar, S. (1990) The ontogeny of the rabbit brain glucose transporter. Endocrinology 126, 2417-2424.

20. Shawver, L. K., Olson, S. A., White, M. K., Weber, M. J. (1987) Degradation and biosynthesis of the glucose transporter protein in chicken embryo fibroblasts transformed by the src oncogene. Mol. Cell Biol. 7, 2112-2118.

21. Sieber, J. E., Traystman, R. J. (1992) Special issues: Glucose and the brain. Critical Care Med. 20, 104-114.

22. Sokoloff, L., Reivich, M., Kennedy, C., Des Rosiers, C., Patlak, H., Pettigrew, K., Sakurada, O., Shinohara, M. (1977) The $\left[{ }^{14} \mathrm{C}\right]$ deoxiglucose method for the measurement of local cerebral glucose utilization: theory, procedure, and normal values in the conscious and anesthetized albino rat. $J$. Neurochem. 28, 897-916.

23. Suda, S., Shinohara, M., Miyaoka, M., Lucignani, G., Kennedy, C., Sokoloff, L. (1990) The lumped constant of the deoxyglucose method in hypoglycemia: effects of moderate hypoglycemia on local cerebral glucose utilization in the rat. J. Cereb. Blood Flow Metab. 10, 499-509.

24. Torizuka, T., Clavo, A. C., Wahl, R. L. (1997) Effect of hyperglycemia on in vitro tumor uptake of tritiated FDG, thymidine, L-methionine and L-leucine, J. Nucl. Med. 38, 382-386.

25. Urabe, T., Hattori, N., Nagamatsu, S., Sawa, H., Mizuno, Y. (1996) Expression of glucose transporters in rat brain following transient focal ishemic injure. J. Neurochem. 67, 265-271.

26. Vannucci, S. J., Seaman, L. B., Vannucci, R. C. (1996) Effect of hypoxia-ischemia on GLUT1 and GLUT3 glucose transporters in immature rat brain. J. Cereb. Blood Flow Metab. 16, 77-81.

27. Wahl, R. L., Henry, C. A., Ethier, S. P. (1992) Serum glucose effects on tumor and normal tissue accumulation of $\left[{ }^{18} \mathrm{~F}\right]$ fluoro-2-deoxy-D-glucose in rodents with mammary carcinoma. Radiology 183, 643-647.

28. Wahl, L. R. (1996) Targeting glucose transporters for tumor imaging: "Sweet" idea, "Sour" result. $J$. Nucl. Med. 37, 1038-104.

29. Walker, P. S., Donovans, J. A., Van Ness, B. G., Fellows, R. E., Pessin, J. E. (1988) Glucose-dependent regulation of the glucose transport activity, protein and mRNA in primary cultures of rat brain glial cells. J. Biol. Chem. 263, 15594-15601. 
